\title{
Salivary uric acid as a noninvasive biomarker of metabolic syndrome
}

\author{
Maria Soukup ${ }^{1}$, Izabela Biesiada ${ }^{1}$, Aaron Henderson² ${ }^{2}$ Benmichael Idowu ${ }^{1}$, Derek Rodeback ${ }^{1}$, Lance Ridpath ${ }^{3}$, \\ Edward G Bridges ${ }^{1}$, Andrea M. Nazar ${ }^{4}$ and Kristie Grove Bridges ${ }^{1 *}$
}

\begin{abstract}
Background: Elevated serum uric acid is associated with obesity, hypertension and metabolic syndrome. Because a linear relationship exists between serum and salivary uric acid (SUA) concentration, saliva testing may be a useful noninvasive approach for monitoring cardiometabolic risk. The goal of this pilot study was to determine if SUA is increased in patients with metabolic syndrome and to investigate correlations between SUA and individual cardiometabolic risk factors.
\end{abstract}

Findings: Volunteers between the ages of 18 and 65 without conditions known to affect serum uric acid levels were recruited. Height, weight, blood pressure and waist circumference were measured and a full lipid panel along with fasting blood glucose was obtained. Saliva samples were collected and uric acid levels were determined. 78 volunteers, $35 \%$ of whom had metabolic syndrome, completed the study. SUA was significantly elevated in patients with metabolic syndrome $(p=.002)$. The incidence of metabolic syndrome in the $4^{\text {th }}$ quartile for SUA was $67 \%$ compared to $25 \%$ in quartiles1-3 combined. Significant correlations were seen between SUA and systolic blood pressure $(r=.440, p=.000)$, diastolic blood pressure ( $r=.304, p=.007)$, waist circumference $(r=.332, p=.003)$, BMI ( $r=.269, p=.018)$, fasting blood glucose $(r=.341, p=.002)$, triglycerides $(r=.410, p=.000), \mathrm{HDL}(r=-.237, p=.036)$ and the number of cardiometabolic risk factors present $(r=0.257, p=.023)$.

Conclusions: These results suggest that SUA may be a useful biomarker for noninvasive monitoring of cardiometabolic risk. Larger studies are needed to validate this approach.

Keywords: Saliva, Biomarker, Metabolic syndrome, Uric acid

\section{Findings}

Introduction

The impact of obesity on health outcomes can be seen in the rising incidence of diabetes and cardiovascular disease, particularly in rural states such as West Virginia (WV). Two-thirds of adults in WV are obese or overweight and over $12 \%$ already have diabetes [1]. In addition, $7.5 \%$ of WV adults have had a heart attack compared to a national average of $4.4 \%$ [1]. Because these diseases can be prevented or delayed by lifestyle interventions, novel approaches to identifying patients at the greatest risk are needed. One tool for identifying high risk patients is screening for metabolic syndrome, a combination of risk factors including increased waist circumference, high

\footnotetext{
* Correspondence: kbridges@osteo.wvsom.edu

${ }^{1}$ Department of Biomedical Sciences, West Virginia School of Osteopathic Medicine, $400 \mathrm{~N}$ Lee St, Lewisburg, WV, USA

Full list of author information is available at the end of the article
}

blood pressure, elevated triglycerides (TRG), reduced high density lipoproteins (HDL), and impaired fasting blood glucose (IFG) [2]. Patients with at least three of these factors are classified as having metabolic syndrome and it is critical that such patients be identified so these modifiable risk factors can be improved.

While the importance of monitoring and reducing cardiometabolic risk is clear, there are significant challenges to addressing this issue, especially in rural areas. These challenges include lack of access, cost, and resistance to invasive monitoring procedures [3]. Noninvasive methods for assessing disease risk could increase participation in screening and treatment programs and improve adherence to dietary and lifestyle interventions. One approach to noninvasive monitoring is the use of salivary biomarkers. Several serum biomarkers associated with cardiometabolic risk including C-reactive protein, adiponectin, and uric acid can also be detected 
in saliva [4-7]. Of particular interest is the fact that a linear relationship between salivary and serum uric acid levels has been observed suggesting that saliva may be a useful surrogate for blood testing [7,8, Soukup et al unpublished results]. While numerous studies have demonstrated an association between serum uric acid, metabolic syndrome and cardiovascular disease risk, its role is not well understood [reviewed in 9,10]. Uric acid is the terminal degradation product of purine catabolism and contributes to the antioxidant capacity of both blood and saliva. However, the enzyme responsible for its production also generates free radicals and several studies have shown that uric acid can act as a pro-inflammatory and pro-oxidant agent [reviewed in 10]. Regardless of whether it plays a causative role or is an indicator of metabolic disturbances, uric acid may be a useful biomarker for identifying high risk patients and monitoring the response to lifestyle interventions. The purpose of this pilot study was to investigate the feasibility of a noninvasive approach by determining if salivary uric acid is elevated in adults with metabolic syndrome.

\section{Study design}

This study (NCT01086137) was approved by the WVSOM Institutional Review Board. Volunteers between the ages of 18 and 65 were recruited from Greenbrier and surrounding counties in WV. Volunteers were not compensated but were given the option to receive blood test results free of charge. Subjects with conditions known to affect salivation, uric acid, or inflammatory biomarkers including gout, renal insufficiency, Sjogren's syndrome, autoimmune disease, active infection, type II diabetes and pregnancy were excluded. A medical history was taken, and information regarding oral health status, family history, medications, supplements and smoking status was collected. Subjects then provided a saliva sample using the passive drool method and flow rates were determined [11]. Height, weight, blood pressure and waist circumference were measured using standard procedures and a full lipid panel along with fasting blood glucose was obtained with a fingerprick sample using the Cholestech LDX instrument [12]. The presence of metabolic syndrome was determined using the new worldwide consensus criteria [13]. Waist circumference cutpoints in these criteria are country specific with US (AHA/NHLBI) guidelines suggesting that the lower values of $\geq 94 \mathrm{~cm}$ in men and $\geq 80 \mathrm{~cm}$ in women are appropriate for populations with an increased likelihood of insulin resistance [13]. Since the majority of subjects in this study were obese or overweight and had a family history of T2DM and nearly $20 \%$ had impaired fasting glucose, these lower cutpoints were used. Analysis using the higher ATPIII cutpoints resulted in re-classification of two subjects and very similar results (data not shown).

\section{Uric acid measurement}

Saliva samples were thawed on ice and the mucins were removed through centrifugation [11]. Supernatants were then applied to a spin column in order to remove peroxidase enzymes that could interfere with the uric acid measurement. Samples contaminated with blood were excluded from analysis. Salivary uric acid (SUA) concentration was determined using the enzymatic uric acid assay reagent

Table 1 Characteristics of the study population and biomarker levels

\begin{tabular}{|c|c|c|c|c|}
\hline & $\begin{array}{c}\text { Total } \\
(n=78)\end{array}$ & $\begin{array}{l}\text { Without Metabolic } \\
\text { Syndrome }(n=51)\end{array}$ & $\begin{array}{c}\text { With Metabolic } \\
\text { Syndrome }(n=27)\end{array}$ & $\bar{p}$ \\
\hline Age & $43.5 \pm 1.8$ & $38.0 \pm 15.5$ & $53.7 \pm 10.2$ & .000 \\
\hline Gender (male/female) & $27 / 51$ & $17 / 34$ & $10 / 17$ & .745 \\
\hline History of periodontal disease (\%) & $21.8 \%$ & $13.7 \%$ & $37.0 \%$ & .018 \\
\hline BMI* & $26.3 \pm 1.2$ & $24.5 \pm 1.2$ & $31.6 \pm 1.1$ & .000 \\
\hline Waist Circumference $(\mathrm{cm})$ & $91.5 \pm 14.3$ & $84.9 \pm 10.6$ & $103.9 \pm 11.9$ & .000 \\
\hline Triglycerides (mg/dL)* & $97.7 \pm 1.8$ & $79.4 \pm 1.5$ & $147.9 \pm 2.0$ & .000 \\
\hline $\mathrm{HDL}(\mathrm{mg} / \mathrm{dL}) *$ & $45.7 \pm 1.4$ & $50.1 \pm 1.3$ & $37.2 \pm 1.3$ & .000 \\
\hline Total Cholesterol (mg/dL) & $189.1 \pm 41.1$ & $176.9 \pm 34.3$ & $212.1 \pm 43.5$ & .000 \\
\hline Glucose (mg/dL) & $91.3 \pm 10.1$ & $88.6 \pm 8.0$ & $96.4 \pm 11.8$ & .001 \\
\hline Systolic BP (mmHg) & $120.2 \pm 13.6$ & $114.8 \pm 11.1$ & $130.4 \pm 12.3$ & .000 \\
\hline Diastolic BP (mmHg) & $78.5 \pm 10.3$ & $74.4 \pm 7.2$ & $86.1 \pm 11.1$ & .000 \\
\hline Salivary Uric Acid ( $\mu \mathrm{M})$ & $217.2 \pm 110.3$ & $184.9 \pm 78.4$ & $278.1 \pm 135.3$ & .002 \\
\hline Salivary flow rate $(\mathrm{mL} / \mathrm{min})^{*}$ & $0.46 \pm 0.72$ & $0.48 \pm 0.47$ & $0.43 \pm 0.53$ & .468 \\
\hline
\end{tabular}

Values are means $+\mathrm{SD}$.

* $\mathrm{P}$ values obtained after $\log _{10}$ transformation. Geometric means are shown. 


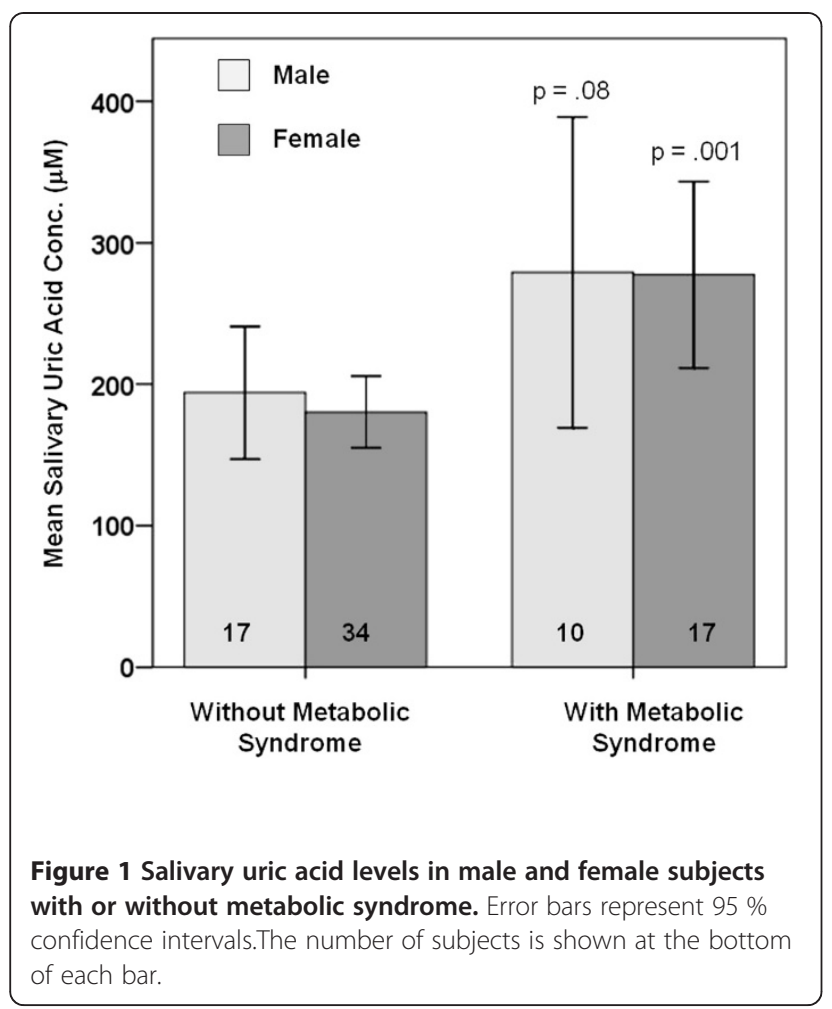

from Pointe Scientific (Canton, MI) in a 96-well format. This method had intra- and inter-assay variability of less than $5 \%$ and average recovery of $104 \%$.

\section{Statistical analysis}

All statistical analyses were performed using PASW version 17 software. Normality was assessed using skewness and kurtosis and values for BMI, salivary flow rate, triglycerides, and serum HDL were normalized with a log transformation. Mean values were compared using unpaired $t$-tests. Categorical values were compared using the chi-squared test. Correlations were investigated by calculating Pearson product-moment correlation coefficients. Spearman's rho was used to investigate correlations between salivary uric acid and the number of cardiometabolic risk factors present. Partial correlations adjusting for sex were also calculated. Statistical significance was set at $\mathrm{p}<0.05$.

\section{Results}

Samples from 78 volunteers who completed the study were included in the analysis. Of these, $35 \%$ had metabolic syndrome. Subject characteristics and biomarker levels are summarized in Table 1. SUA levels were significantly elevated in patients with metabolic syndrome $(p=.002)$ independent of salivary flow rate. No significant differences were seen between overall SUA levels in men vs. women, or people with a history of periodontal disease vs. those without. However, the relationship between SUA and metabolic syndrome was stronger in women $(\mathrm{p}=.001)$ than in men $(\mathrm{p}=.08)$ (Figure 1$)$. The overall incidence of metabolic syndrome in the $4^{\text {th }}$ quartile for SUA was $67 \%$ compared to $18-33 \%$ in quartiles $1-3$ (Figure 2). Significant positive correlations were seen between SUA and systolic blood pressure $(\mathrm{r}=.440, \mathrm{p}=.000)$, diastolic blood pressure $(\mathrm{r}=.304, \mathrm{p}=.007)$, waist circumference $(\mathrm{r}=.332, \mathrm{p}=.003)$, BMI $(\mathrm{r}=.269, \mathrm{p}=.018)$, fasting blood glucose $(\mathrm{r}=.341, \mathrm{p}=.002)$, triglycerides $(\mathrm{r}=.410$, $\mathrm{p}=.000)$ and the number of cardiometabolic risk factors present $(\mathrm{r}=0.257, \mathrm{p}=0.023)$. SUA levels were negatively

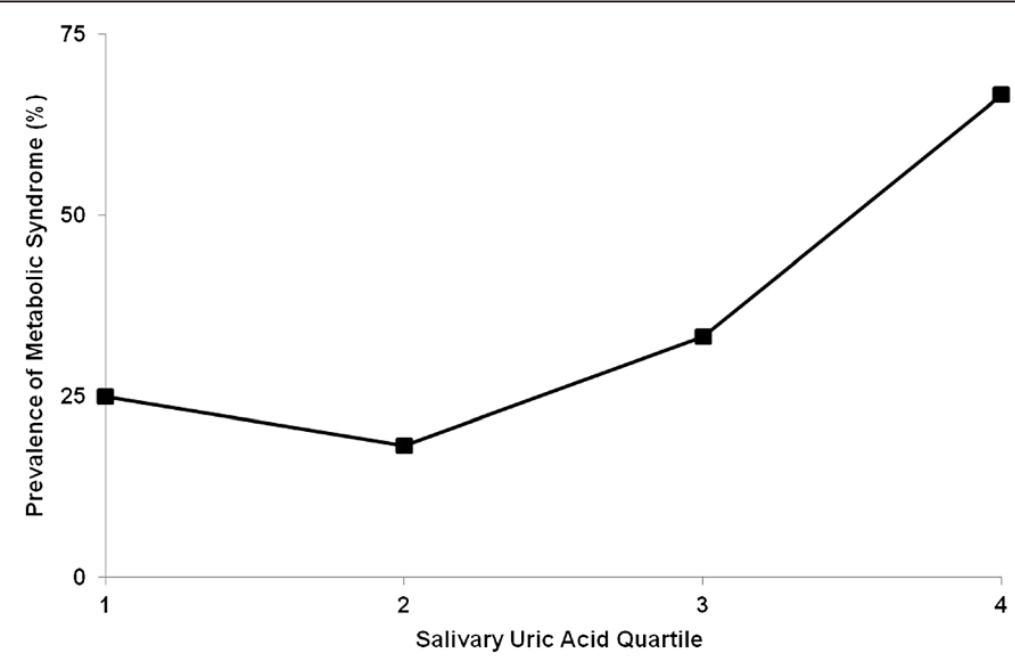

Figure $\mathbf{2}$ The prevalence of metabolic syndrome by uric acid quartile. The number of subjects in quartiles one through four were $20,22,18$ and 18 respectively. 
correlated with HDL ( $\mathrm{r}=-.237, \mathrm{p}=.036)$. These relationships remained significant when partial correlations were calculated after adjusting for sex.

\section{Discussion}

In recent years, significant advances have been made toward the validation of salivary biomarkers for disease detection. Methods using oral fluids for diagnosis of disorders including myocardial infarction, periodontal disease, and cancer are being investigated [14]. Because both metabolic syndrome and T2DM have been linked to periodontal disease, oral health care providers have an important role in identifying high risk patients, making oral diagnostics a good fit for these indications. Several studies have shown that serum uric acid levels are elevated in patients with metabolic syndrome [reviewed in 9, 10]. This study demonstrates that salivary uric acid is also elevated in patients with metabolic syndrome and correlates with several cardiometabolic risk factors including blood pressure, triglyceride levels, HDL and fasting blood glucose. As seen in other studies looking at serum, the relationship between SUA and metabolic syndrome was stronger in females than in males [15-17]. While larger studies are needed to delineate the factors contributing to SUA levels and their association with metabolic syndrome, our results suggest that that SUA may be a viable noninvasive biomarker for monitoring cardiometabolic risk particularly in women.

Limitations of this pilot study include the small sample size, the fact that oral health status was selfreported, and the absence of detailed information regarding emotional status, nutrition and alcohol consumption which could affect salivation or uric acid levels. However, the results suggest that this approach should be further explored. Follow-up studies to validate SUA as a biomarker of metabolic syndrome should determine not only its predictive value but also its ability to improve models consisting of other noninvasive measures such as waist circumference. The inclusion of additional salivary biomarkers might further improve these models and studies investigating other salivary changes associated with obesity and metabolic syndrome have recently been published $[18,19]$. These candidate markers could be included in future studies. The ability to monitor cardiometabolic risk using simple, noninvasive testing should help overcome barriers to screening and may also improve adherence to dietary and behavioral treatment programs.

Competing interests

The authors declare that they have no competing interests.

\section{Acknowledgements}

The authors thank Jason Reed, Rafal Kopanczyk, Jackie Lickliter, Daniel Opris, Michelle Vanoy-Warner and the WVSOM intramural grant program.

\section{Author details}

${ }^{1}$ Department of Biomedical Sciences, West Virginia School of Osteopathic Medicine, 400 N Lee St, Lewisburg, WV, USA. ${ }^{2}$ Current affiliation, Department of Internal Medicine, San Antonio Uniformed Services Health Education Consortium, San Antonio, TX, USA. ${ }^{3}$ Department of Assessment and Educational Development, West Virginia School of Osteopathic Medicine, Lewisburg, WV, USA. ${ }^{4}$ Department of Clinical Sciences, West Virginia School of Osteopathic Medicine, Lewisburg, WV, USA.

\section{Authors' contributions}

MS assisted with data collection and analysis, completed the biomarker assays, and drafted the manuscript. IB, BI and DR assisted with data collection and uric acid analysis. LR participated in the study design and completed the statistical analysis. AH, EB and AN assisted with study design and coordination, drafting of the clinical protocol, and data collection and analysis. KB conceived of the study, assisted in its design, coordination, and analysis, and helped to draft the manuscript. All authors read and approved the final manuscript.

Received: 14 February 2012 Accepted: 19 April 2012

Published: 19 April 2012

\section{References}

1. 2006 West Virginia Behavioral Risk Factor Survey Report [http://www. wvdhhr.org/bph/HSC/Pubs/BRFSS/2006/default.htm]

2. Kassi E, Pervanidou P, Kaltsas G, Chrousos G: Metabolic syndrome: definitions and controversies. BMC Med 2011, 9:48.

3. Deskins S, Harris CV, Bradlyn AS, Cottrell L, Coffman JW, Olexa J, Neal W: Preventive care in Appalachia: use of the theory of planned behavior to identify barriers to participation in cholesterol screenings among West Virginians. J Rural Health 2006, 22:367-374.

4. Mamali I, Roupas ND, Armeni AK, Theodoropoulou A, Markou KB, Georgopoulos NA: Measurement of salivary resistin, visfatin and adiponectin levels. Peptides 2012, 33:120-124.

5. Ouellet-Morin I, Danese A, Williams B, Arseneault L: Validation of a highsensitivity assay for C-reactive protein in human saliva. Brain Behav Immun 2011, 25:640-646.

6. Dillon MC, Opris DC, Kopanczyk R, Lickliter J, Cornwell HN, Bridges EG, Nazar AM, Bridges KG: Detection of homocysteine and C-reactive protein in the saliva of healthy adults: comparison with blood levels. Biomark Insights 2010, 5:57-61.

7. Goll RD, Mookerjee BK: Correlation of biochemical parameters in serum and saliva in chronic azotemic patients and patients on chronic hemodialysis. J Dial 1978, 2:344-399.

8. Nunes LA, Brenzikofer R, Macedo DV: Reference intervals for saliva analytes collected by a standardized method in a physically active population. Clin Biochem 2011, 44:1440-1444

9. Nakagawa T, Cirillo P, Sato W, Gersch M, Sautin Y, Roncal C, Mu W, SánchezLozada LG, Johnson RJ: The conundrum of hyperuricemia, metabolic syndrome, and renal disease. Intern Emerg Med 2008, 3:313-318.

10. Lippi G, Montagnana M, Franchini M, Favaloro EJ, Targher G: The paradoxical relationship between serum uric acid and cardiovascular disease. Clin Chim Acta 2008, 392:1-7.

11. Henderson AT, Fisher JF, Blair J, Shea C, Li TS, Bridges KG: Effects of rib raising on the autonomic nervous system: a pilot study using noninvasive biomarkers. J Am Osteopath Assoc 2010, 110:324-330.

12. Centers for Disease Control and Prevention. The Third National Center for Health Statistics: National Health and Nutrition Examination Survey (NHANES III 1988-94) reference manuals and reports National Center for Health Statistics, Bethesda, MD; 1996.

13. Alberti KG, Eckel RH, Grundy SM, Zimmet PZ, Cleeman II, Donato KA, Fruchart JC, James WP, Loria CM, Smith SC: Harmonizing the metabolic syndrome: a joint interim statement of the International Diabetes Federation Task Force on Epidemiology and Prevention; National Heart, Lung, and Blood Institute; American Heart Association; World Heart Federation; International Atherosclerosis Society; and International Association for the Study of Obesity. Circulation 2009, 120:1640-1645.

14. Giannobile WV, McDevitt JT, Niedbala RS, Malamud D: Translational and clinical applications of salivary diagnostics. Adv Dent Res 2011, 23:375-380.

15. Rodrigues SL, Baldo MP, Capingana P, Magalhães P, Dantas EM, Molina MD, Salaroli LB, Morelato RL, Mill JG: Gender distribution of serum uric acid 
and cardiovascular risk factors: a population based study. Ara Bras Cardiol 2012, 98:13-21.

16. Yang T, Chu CH, Bai CH, You SL, Chou YC, Chou WY, Chien KL, Hwang LC, Su TC, Tseng CH, Sun CA: Uric acid level as a risk marker for metabolic syndrome: a Chinese cohort study. Atherosclerosis 2012, 220:525-531.

17. Chiou WK, Wang MH, Huang DH, Chiu HT, Lee YJ, Lin JD: The relationship between serum uric acid level and metabolic syndrome: differences by sex and age in Taiwanese. J Epidemiol 2010, 20:219-224.

18. Tremblay M, Loucif Y, Methot J, Brisson D, Gaudet D: Salivary pH as a marker of plasma adiponectin concentrations in women. Diabetol Metab Syndr 2012, 4:4.

19. Qvarnstrom M, Janket SJ, Jones JA, Jethwani K, Nuutinen P, Garcia RI, Baird AE, Van Dyke TE, Meurman JH: Association of salivary lysozyme and C-reactive protein with metabolic syndrome. J Clin Periodontol 2010, 37:805-811.

doi:10.1186/1758-5996-4-14

Cite this article as: Soukup et al: Salivary uric acid as a noninvasive biomarker of metabolic syndrome. Diabetology \& Metabolic Syndrome 2012, 4:14.

\section{Submit your next manuscript to BioMed Central and take full advantage of:}

- Convenient online submission

- Thorough peer review

- No space constraints or color figure charges

- Immediate publication on acceptance

- Inclusion in PubMed, CAS, Scopus and Google Scholar

- Research which is freely available for redistribution 